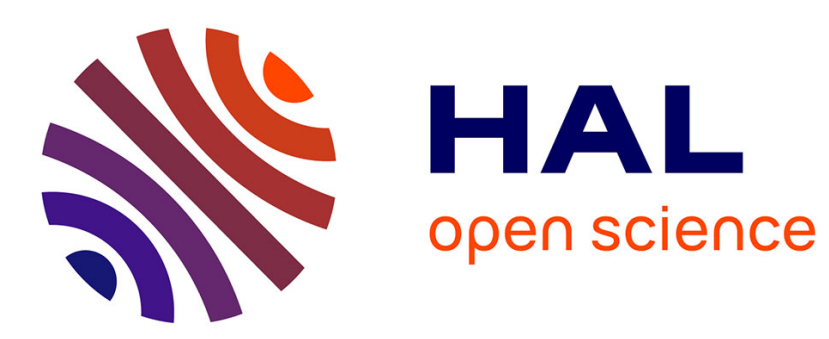

\title{
La relation banque-entreprise: structures de gouvernement et formes de coordination
}

Jean-Pierre Allegret, Bernard Baudry

\section{To cite this version:}

Jean-Pierre Allegret, Bernard Baudry. La relation banque-entreprise : structures de gouvernement et formes de coordination. Revue Française d'Economie, 1996, 11 (4), pp.3-36. hal-01660199

\section{HAL Id: hal-01660199 \\ https://hal.science/hal-01660199}

Submitted on 15 May 2018

HAL is a multi-disciplinary open access archive for the deposit and dissemination of scientific research documents, whether they are published or not. The documents may come from teaching and research institutions in France or abroad, or from public or private research centers.
L'archive ouverte pluridisciplinaire HAL, est destinée au dépôt et à la diffusion de documents scientifiques de niveau recherche, publiés ou non, émanant des établissements d'enseignement et de recherche français ou étrangers, des laboratoires publics ou privés. 


\section{LA RELATION BANQUE-ENTREPRISE : STRUCTURES DE GOUVERNANCE ET FORMES DE COORDINATION *}

Jean-Pierre Allegret - Maître de Conférences

Centre de Recherche Monnaie-Finance-Banque

Bernard Baudry - Maître de Conférences

Economie des Changements Technologiques (URA CNRS)

Université Lumière Lyon 2

* Une version préliminaire de ce texte a été présentée à la Table ronde "Finance et industrie" de l'atelier "Macroéconomie Financière" du GDR Monnaie et Financement (Lyon, 24-25 mars 1995). Nous remercions l'ensemble des participants pour leurs commentaires. Nos remerciements vont aussi à Patrice GEOFFRON (Université de Reims) pour l'attention qu'il a apporté à notre travail. 
RESUME

\section{LA RELATION BANQUE-ENTREPRISE : STRUCTURES DE GOUVERNANCE ET FORMES DE COORDINATION}

Pour analyser les structures de gouvernance potentielles entre les banques et les entreprises, les auteurs étudient la pertinence du concept de quasi-intégration pour caractériser la relation banque/entreprise. Trois formes pures de gouvernance banque/entreprise sont ainsi mises en évidence, le marché, la quasi-intégration et la hiérarchie. Des éléments descriptifs empruntés aux modèles allemands et japonais permettent de caractériser ces structures de gouvernance. Si, dans une relation de marché, l'incitation à court terme constitue la forme de coordination principale, dans la relation hiérarchique, c'est l'autorité. Enfin, dans la relation de quasi-intégration, trois formes de coordination se combinent, l'autorité, l'incitation et la confiance. Compte tenu de cette pluralité, la quasi-intégration constitue une forme organisationnelle efficiente, notamment pour les PME confrontées à l'incertitude et à la mutation des marchés.

\section{ABSTRACT \\ BANK-FIRM RELATIONSHIPS : GOVERNANCE STRUCTURES AND FORMS OF COORDINATION}

To analyse governance structures between banks and firms, the authors test the relevance of the concept of "quasi-integration" use in industrial economics to indicate an intermediate transaction between market and hierarchy. The criterion of information is here essential to study the relation between firms and banks. In a quasi-integration relationship, first information is shared and generates sunk costs, second the relation is a long term one, and last such a relation allow the firm to invest in innovative projects. Whereas in a market relationship, incentive is the principal form of coordination, in a hierarchy relationship, it is in fact authority. In the second part of the paper, we show that in a quasi-integration relationship, three forms of coordination are combined together : authority, incentive and trust. Because of this plurality, such governance structure leads to efficiency, by reducing internal uncertainty that burdens the transaction between banks and firms, and by promoting adaptability and flexibility when the environment is changing. 


\section{Introduction}

L'analyse des systèmes de financement et la relation banque-entreprise occupent une place croissante dans la littérature économique. Deux tendances significatives émergent de cette littérature.

Un premier groupe d'analyses se développe autour des avantages relatifs de tel ou tel type de financement. Cette littérature est particulièrement influencée par la partition traditionnelle entre les systèmes anglo-saxons fondés sur les marchés et les systèmes germano-japonais fondés sur le crédit. La diversité des conclusions démontre qu'il n'existe pas à l'heure actuelle de consensus sur le type de financement optimal du point de vue de la croissance des entreprises. Ainsi, certains auteurs soulignent les vertus du financement interne par rapport au financement externe (Gertner, Stein et Scharfstein (1994) : accroissement de l'incitation à la surveillance dans la mesure où l'investisseur est aussi le propriétaire des entreprises à financer ; plus grande possibilité de redéployer les actifs dans la mesure où l'investisseur possède en fait un portefeuille d'unités de production. Du point de vue du seul financement externe, Allen (1993) souligne les gains attendus des financements décentralisés (les marchés financiers) par opposition aux financements centralisés (recours à un nombre réduit de banques). L'axe essentiel de sa démonstration repose sur la capacité des marchés à faire émerger des opinions divergentes sur les stratégies optimales, source de gains d'efficience dans les activités où les évolutions sont rapides. Enfin, Goodhart (1994) et Benston (1994) s'attachent à démontrer les avantages des systèmes de banque universelle qui inscrivent les relations emprunteurs-créditeurs dans la durée et la stabilité.

Un second groupe d'analyses s'est développé à partir des théories microéconomiques de l'intermédiation qui raisonnent dans un cadre d'asymétrie informationnelle. Ces analyses mettent l'accent sur les procédures d'incitation mises en oeuvre par les intermédiaires financiers afin de faire face aux problèmes de risque moral. Au coeur de cette approche se situe la capacité des intermédiaires financiers à capter les informations pertinentes dans un univers incertain et leur capacité relativement aux actionnaires- à contrôler efficacement les emprunteurs (Stiglitz, 1985). Les caractéristiques du contrat de dette découlent directement de cette observation. Ainsi, Gorton et Kahn (1993) démontrent la capacité de l'intermédiaire financier -prêteur unique- à mettre en oeuvre des procédures d'incitations plus crédibles que si le financement était effectué par de multiples prêteurs (cas des marchés d'actions et d'obligations). En effet, le prêteur unique peut spécifier par contrat un certain nombre d'engagements assumés par l'emprunteur qui forceront ce dernier à rembourser sa dette. De leur côté, Aghion et Bolton (1992) démontrent le fait que dans un monde où les contrats sont incomplets, seul le contrat de dette 
permet d'établir une incitation optimale de l'emprunteur en spécifiant les procédures de contrôle de la propriété selon les résultats obtenus par cet emprunteur.

La présente contribution s'inscrit dans le cadre de l'étude des caractéristiques de la relation banque-entreprises en accordant une attention particulière aux questions de l'incitation et du contrôle. Plus précisément, .elle vise à tester la pertinence du concept de quasi-intégration, concept emprunté à l'économie industrielle (Houssiaux, 1957) pour qualifier une relation non strictement marchande et non internalisée, pour analyser la relation banque-entreprise et à souligner sa spécificité.

Une double perspective est adoptée. D'une part, une perspective normative qui vise à établir les conditions d'existence de la quasi-intégration et ses conditions d'efficience telles qu'elles peuvent être identifiées lors de l'étude de la relation banque-entreprise. A ce niveau, la question de l'information occupe une place centrale, ce qui différencie nettement le recours au modèle marchand en tant que structure de gouvernance de la quasi-intégration. Ainsi, en suivant Williamson (1988), nous considérons les modalités de financement comme des structures de gouvernance. D'autre part, une perspective positive qui a pour objectif de souligner l'importance de la quasi-intégration pour faire face, du point de vue du financement long des entreprises (et plus particulièrement des PME), aux effets déstabilisants de la globalisation financière.

Nous montrons dans un premier temps que les structures de gouvernance potentielles des relations banque/entreprise sont comparables à celles qui régulent les transactions inter-firmes (organisation industrielle), puisque dans les deux cas, trois structures pures peuvent être identifiées, le marché, la quasi-intégration, et la hiérarchie.

Trois formes de coordination soutiennent la relation de quasi-intégration, l'autorité, l'incitation et la confiance, et permettent de réduire l'incertitude inhérente à la relation banque/entreprise. En étudiant la combinaison de ces trois formes, nous montrons alors dans un deuxième temps que dans un environnement incertain, la structure de gouvernance qualifiée de quasi-intégration correspond, comme sur le marché des produits, à une configuration optimale au niveau de la circulation de l'information(1).

\section{I/ Incomplétude contractuelle et "structures de gouvernance" potentielles des relations banque-entreprise}

Lorsque l'on postule une hypothèse de rationalité limitée, le contrat conclu entre une banque et une entreprise se révèle incomplet $(A /)$; trois formes pures de 
structures de gouvernance sont alors susceptibles de réguler la relation banque/entreprise : le marché, la hiérarchie et la quasi-intégration ( $B /$ ).

\section{A/ Qualification de l'objet échangé et incomplétude contractuelle}

Dans les relations banque-entreprise et inter-entreprises, les objets de la coordination -souscription d'un emprunt d'une part, achat d'un produit industriel spécifique d'autre part, nous semblent en partie comparables.

Lorsqu'une entreprise sollicite un prêt, elle vend de l'information et cette vente lui permet de se procurer des fonds. En ce sens, de la part de la banque, le prêt correspond bien à un pari sur l'avenir, puisqu'en effet, rien ne garantit ex post la possibilité de remboursement du prêt.

En échange de l'information obtenue, la banque acquiert une créance qui doit lui permettre de recouvrer les fonds initiaux, recouvrement assorti d'un intérêt. Dès lors, une variable nous paraît essentielle pour envisager la relation banque/entreprise, la qualité de l'information cédée par l'entreprise. Cette qualité recouvre en réalité deux éléments :

- la qualité matérialisée par les garanties que l'entreprise est en mesure de fournir à la banque (garanties en termes de stock).

- la qualité du projet d'investissement envisagé (garanties en termes de flux de revenus futurs).

Cette spécificité du contrat banque/entreprise a été décrite avec précision par certains auteurs. Cette littérature se fonde sur les problèmes informationnels entre débiteurs et créanciers. En effet, cette information -cruciale pour la banque- n'est pas partagée également. Comme sur le marché des produits, deux sources essentielles d'asymétrie informationnelle coexistent :

- l'anti-sélection : comme sur le marché des voitures d'occasion analysé par Akerlof en 1970, le marché du crédit a toutes les chances d'être déserté par les entreprises qui possèdent la meilleure "qualité informationnelle". En effet, comme la banque ne connaît pas ex ante cette qualité, elle aura tendance à exiger un prix élevé pour l'acquisition d'une créance, ne restant alors sur le marché que les entreprises qui ne peuvent trouver des fonds par d'autres canaux.

- le risque moral : ex post, il existe un risque de défaillance de l'entreprise si l'investissement financé par le prêt bancaire accroît la probabilité de non remboursement. Dans ce deuxième cas de figure, la banque ne sait pas si l'entreprise agira dans l'intérêt de son créancier, c'est-à dire si elle utilisera de manière optimale le crédit octroyé. 
Dans ces conditions, la banque ne pourra octroyer les fonds aux investisseurs ayant les opportunités d'investissement les plus productives, et ce d'autant plus que l'investissement en question sera risqué. Cette situation sera de plus aggravée si l'environnement externe est marqué par une incertitude forte.

Cette analyse est en tout point comparable à ce que l'on constate sur le marché des produits industriels spécifiques (Baudry, 1995) : ex ante, l'acheteur ne peut pas évaluer les capacités du vendeur à livrer un bien répondant à certaines spécifications techniques complexes, et, ex post, lorsque la relation est à moyen terme, l'acheteur est dans l'impossibilité de relier le paiement du fournisseur en fonction du niveau d'effort engagé par ce dernier.

Finalement, dans les deux types de relation, lorsque l'on retient l'hypothèse comportementale de rationalité limitée, un contrat explicite complet garanti par la loi ne peut lever l'incertitude.

Compte tenu de cette nature particulière de l'objet transacté, quelles sont alors les formes organisationnelles susceptibles de réguler la relation banque/entreprise?

B/ La mise en évidence de trois formes pures de structures de gouvernance : le marché, la hiérarchie et la quasi-intégration

A priori, comme sur le marché des produits (relations inter-firmes), il est possible d'envisager trois formes pures de relations banque/entreprise, trois formes potentielles de structures de gouvernance : le marché, la quasi-intégration, la hiérarchie. Deux critères mettent en évidence ces trois formes : la durée de la relation d'une part, le degré de partage de l'information contrôle d'autre part, et ce en retenant un niveau d'incertitude moyen. 


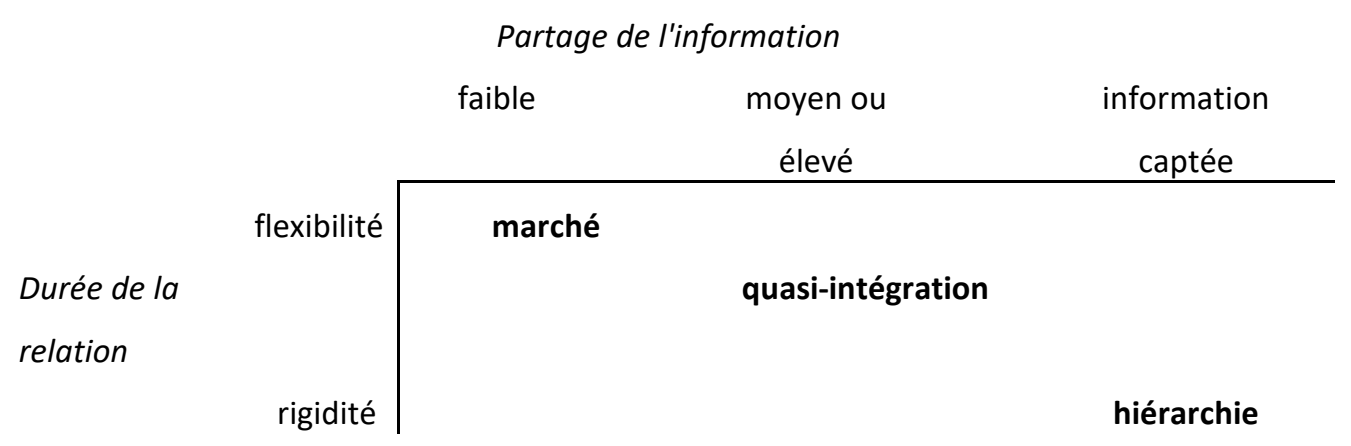

Dans un premier temps, nous nous intéresserons au marché et à la hiérarchie, en indiquant les formes de coordination correspondantes, puis nous analyserons de manière plus substantielle l'objet retenu dans ce travail, c'est-à-dire la quasiintégration.

\section{1 / La structure de gestion par le marché}

Ce qui caractérise cette configuration, c'est d'une part la flexibilité de la relation, et, d'autre part, l'absence de contrôle de la banque sur la qualité de l'information possédée par l'entreprise, l'information étant donc ici non partagée.

Dans ce type de relation, les acteurs ne s'engagent pas dans une relation durable, pour conserver une liberté de choix quant aux opportunités futures : c'est donc la réversibilité et la "défiance" (Rivaut-Danset et Salais, 1992) qui caractérisent ce qui n'est en définitive qu'un "pseudo-engagement". D'une certaine manière, ce type de contrat rappelle le contrat qualifié par Williamson (1975) de "sequential spot contract", à propos du marché des produits intermédiaires. Ce type de contrat consiste à "attendre jusqu'à ce que le futur se matérialise et (à) contracter alors au moment de l'apparition des évènements"(2). Alors que des "contingent claims contracts" sont impossibles à établir du fait de la rationalité limitée et de l'opportunisme, les "contrats séquentiels à court terme" sont uniquement contraints par l'opportunisme. En effet, aucun effort n'est entrepris pour décrire au départ (en $t_{0}$ ) un arbre de décision complet. Au contraire, du fait que l'adaptation à l'incertain s'effectue au fur et à mesure que des évènements nouveaux surviennent, il a l'avantage d'économiser sur la rationalité limitée.

Autrement dit, ce type de contrat possède deux avantages pour les contractants

- lorsque l'incertitude environnementale est forte, un contrat à court terme, flexible, permet la réversibilité de l'engagement : la banque comme l'entreprise peuvent avoir intérêt à court terme à cesser la relation, en cas d'irruption d'événements non anticipés en $t_{0}$. 
- d'autre part, un contractant dupé par un comportement opportuniste de l'autre contractant (non respect des engagements initiaux) a la faculté de sortir rapidement de la relation, ce désengagement n'entraînant pas de surcroît des coûts irrécupérables (inexistence de "sunk costs").

Dans ce modèle, il y a donc absence de fidélité vis-à-vis de l'autre : la banque diversifie sa clientèle, et l'entreprise diversifie ses sources de financement. Ce modèle correspond à ce que les économistes nomment "banque à l'acte" ; la relation est purement contractuelle et limitée dans le temps, les partenaires minimisent le coût de construction de l'information et activent à intervalles réguliers la concurrence.

Néanmoins, ce modèle marchand présente selon nous deux désavantages principaux.

Premièrement, si l'on analyse ce mode d'engagement en termes de théorie des jeux, on se rend compte qu'un tel modèle débouche sur la situation classique du dilemme du prisonnier et sur une stratégie non coopérative : les agents activent la concurrence à intervalles de courte durée et il y a défiance généralisée : l'entreprise a peur qu'en dévoilant la véritable qualité de son information, la banque se désengage, et de son côté, la banque n'a pas intérêt à se montrer coopérative (c'est-à-dire accorder des prêts alors qu'elle ne connaît pas la qualité réelle de l'information qu'elle acquiert) si elle anticipe une rupture rapide de la relation. En ce sens, un tel modèle s'apparente à un jeu à un seul coup, ce qui conduit à une solution sous-optimale pour les deux contractants : la banque ne peut fidéliser ses débiteurs, et l'entreprise ne peut pas s'engager dans des investissements qui présentent un certain degré de risque. En effet, comme l'entreprise a une propension à dissimuler la qualité de l'information qu'elle vend, la banque tend à adopter un comportement prudentiel en matière d'octroi de prêts. Pour reprendre la terminologie célèbre de Hirschman (1972) sur les réactions possibles des acteurs face à des difficultés, ces derniers optent ici clairement pour la stratégie de l'exit.

Secondement, du fait d'une relation courte, au moins contractuellement, cette structure de gouvernance n'autorise pas un apprentissage relationnel source de richesses futures.

Dans cette structure de gouvernance, la forme principale de coordination est clairement l'incitation contenue dans le contrat de courte période. La problématique de la relation entre l'intermédiaire financier et l'entreprise se réduit aux termes suivants : à $t_{0}$ : obtention du prêt; à $t_{1}$ : observation d'une situation de défaut (faillite) ou de non défaut (non faillite) ${ }^{(3)}$.

L'objectif est alors d'inciter les débiteurs à la situation de non défaut. La solution proposée est la menace d'exclusion à l'instant to et l'exclusion effective selon l'observation de l'état à $t_{1}$. II n'apparaît donc pas dans ce modèle marchand de 
représentation explicite d'un processus d'apprentissage pouvant conduire les acteurs à modifier leur stratégie(4).

Stiglitz et Weiss (1983) élaborent un modèle dans lequel la menace est celle de la rupture du contrat de prêt à $t_{1}$. Selon les auteurs, les "contrats contingents ont certaines propriétés désirables d'incitation"(5). Les états contingents sont le défaut et le non défaut, notés respectivement $s_{11}$ et $s_{12}$. Soit alors : $t_{0}$ obtention du prêt et $t_{1}$

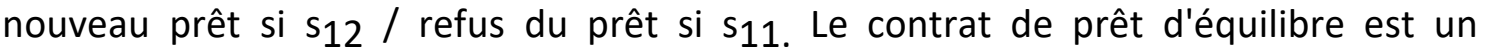
équilibre de Nash. Les caractéristiques du contrat qui maximisent les rendements de la banque sont les suivantes:

- la disponibilité du prêt à $t_{1}$ est contigente à la situation de non défaut $d u$ débiteur sur les dettes de $t_{0}$;

- l'obtention éventuelle d'un prêt est assise sur les performances obtenues à to. Les entreprises en situation de non paiement supportent des taux d'intérêt plus élevés que les autres. Dès lors, en raison des phénomènes de sélection adverse, leur exclusion est probable.

Le modèle de Allen (1983) se situe dans la lignée du précédent mais il a la volonté de rejeter l'exogénéité des coûts de défaut. Dans cette perspective, "les principales pénalités pour un défaut sont imposées par le marché lui-même : les emprunteurs qui n'effectuent pas de paiements sur leurs prêts sont exclus du marché"(6). En effet, pour que la menace soit crédible, il faut qu'il y ait une exclusion effective du marché du capital.

Des modèles d'incitation multipériodiques se sont développés dans le cadre de ce que nous appelons la relation marchande. Ainsi, Haubrich (1989) présente un modèle d'intermédiation dans lequel la durée de la relation débiteur-créancier permet à l'intermédiaire d'établir un test statistique issu des relations passées, test qui lui permet d'éliminer les mauvais dossiers. Webb (1989) met en avant les avantages tirés de l'observation répétée sur plusieurs périodes, les intermédiaires financiers étant mieux à même d'éliminer les emprunteurs défaillants en faisant peser sur eux la menace d'une dégradation des conditions de prêts. Autrement dit, le contrat incitateur optimal sur deux périodes est celui qui conditionne les termes du paiement aux résultats de la période 1 . La dimension intertemporelle de ce type d'intermédiation permet de réduire les coûts d'information et de mieux sélectionner les bons emprunteurs.

La signification de ces modèles nous paraît devoir être soulignée : alors qu'ils sont couramment considérés comme étant représentatifs d'une relation de clientèle cette caractérisation trouvant appui sur la durée de la relation- ces modèles relèvent en fait dans notre perspective de la logique de l'exit, et par là-même de la pure relation marchande. 


\section{$\underline{2 / \text { La structure de gestion par la hiérarchie }}$}

A l'opposé de ce modèle, on trouve la structure de gouvernance unifiée. Dans ce type de relation, la banque décide de prendre le contrôle de l'entreprise. Un tel mode de gouvernance permet à la banque d'acquérir l'information qui lui fait défaut dans une relation marchande. Sur le marché des produits intermédiaires, cette solution est envisagée par Williamson (1975) lorsque le risque lié à l'opportunisme de la part du fournisseur est maximal. Dans ce cas, le recours à la hiérarchie, par l'intermédiaire de l'autorité, permet de supprimer l'opportunisme du vendeur(7).

Dans le spectre des relations banque-entreprise présentées précédemment, le modèle japonais de la banque principale nous paraît incarner cette structure de gestion par la hiérarchie.

La structure de financement des entreprises est ici marquée par la dépendance de la firme à l'égard des prêts bancaires. De par la diversité des services offerts par les banques - gestion de trésorerie, obtention de crédits à court et long termes, chef de file des syndicats bancaires - la banque principale est en mesure de suivre quotidiennement l'activité de la firme. Le suivi des comptes - par la gestion de trésorerie - et l'étude des projets - lors d'une demande de fonds - permettent à la banque principale de stocker des informations tant conjoncturelles que structurelles concernant la situation de la firme.

La structure de propriété de l'économie japonaise se caractérise par un actionnariat stable à deux composantes. La première est faite de participations croisées entre firmes du groupe qui induit une limitation importante de la circulation des titres sur le marché boursier. La seconde composante est celle des intermédiaires financiers, véritable épicentre du système, qui possèdent dans leur portefeuille des titres de propriétés des entreprises concernées et entretiennent un réseau de participations croisées avec les firmes du groupe. Autrement dit, la banque principale appartient à la fois au groupe des créanciers et à celui des actionnaires.

La structure de gouvernance de la relation banque-entreprises au sein du système japonais est la résultante de cette double appartenance de la banque. L'échange d'informations entre les différents protagonistes intervient à tous les stades de la conception et de la réalisation d'un projet productif. Si le Conseil d'Administration gère les activités au quotidien de la firme sans que la banque principale y joue habituellement un rôle central, le Conseil des Présidents -club informel dont les réunions sont mensuelles- apparaît comme le lieu stratégique dans lequel s'exprime le pouvoir de la banque actionnaire-créancière. Prowse note à ce propos qu'il "constitue une sorte de tribune où les partenaires financiers et les dirigeants discutent de la politique de l'entreprise"(8). Cela signifie que le contrôle de la 
banque ne s'exerce qu'indirectement sur l'entreprise, soit par l'intermédiaire de son activité de prêts, soit par le mécanisme du Conseil des Présidents.

Cet aspect indirect de la surveillance bancaire nous conduit d'ailleurs à caractériser de hiérarchique la structure de gestion du modèle japonais. Au sein de ce modèle, la banque contient l'opportunisme de l'entrepreneur-débiteur en impulsant un double mécanisme à base d'autorité :

- d'une part, un mécanisme structurant -que nous qualifierons de mécanisme social- dans lequel le positionnement hiérarchique des acteurs joue comme force de rappel pour garantir l'efficacité du comportement entrepreneurial. La perspective de promotion hiérarchique ou de non promotion -le promotional hierarchies of ranks de Aoki et Dosi (1992)- codifie strictement la signification de la surveillance bancaire des activités des managers ;

- d'autre part, le mécanisme de la sanction ultime dans lequel la banque principale prend le contrôle de l'entreprise en cas de difficultés structurelles. La conséquence de la prise de contrôle est le remplacement de l'équipe managériale.

Ainsi, la gestion par la hiérarchie dans le modèle japonais implique une connaissance précise de la sanction -le contrôle par la banque-actionnaire- et une compréhension immédiate de cette sanction par le jeu des représentations sociales. Cette relation hiérarchique s'exprime aussi par le caractère unilatéral de la participation de représentants de la banque au Conseil d'Administration : si les banques envoient leurs employés dans les entreprises, l'inverse n'est pas vrai alors même que des participations croisées existent (Hoshi, 1994).

Les limites de la gestion par la hiérarchie sont loin d'être négligeables. Premièrement, la contrainte du changement rapide semble peu compatible avec la relative rigidité des relations banque-entreprise. Le mode organisationnel qui s'impose est porteur de procédures routinières contraires à sa flexibilité nécessaire ${ }^{(9)}$. Deuxièmement, l'intensité des participations croisées rend opaque la gestion des firmes qui appartiennent au même groupe. II en résulte une difficulté endémique source d'inefficiences allocatives- à discipliner l'usage du free cash flow. Troisièmement enfin, le modèle japonais se caractérise par l'absence d'un véritable contre-pouvoir à la banque principale. Ce dernier aspect constitue la pierre angulaire de la gestion par la hiérarchie dans laquelle il ne s'établit pas de véritables relations partenariales entre les acteurs. A ce niveau, comme il apparaîtra par la suite, la différence avec le modèle allemand - qui relève en partie de la quasi-intégration - est extrêmement importante du point de vue de la pérennité et de l'efficacité du système. Les récentes mutations du système financier japonais, dont la conséquence est un élargissement des canaux de financement et par là-même une moindre dépendance au financement bancaire, ont été l'occasion pour les grandes entreprises 
d'amoindrir le pouvoir des organismes bancaires et ce en dépit des avantages économiques du système soulignés précédemment ${ }^{(10)}$.

\section{3/ La quasi-intégration}

Dans le champ de l'économie industrielle, le concept de quasi-intégration a été introduit en France par Houssiaux (1957) pour désigner la relation de sous-traitance. La quasi-intégration désigne une zone intermédiaire entre le marché et l'intégration, et "qui comprend les relations habituelles de clientèle entre des firmes situées à des stades de production successifs" (11). Cette analyse en termes de quasi-intégration a ensuite été largement reprise pour appréhender les nouvelles formes d'organisation inter-entreprises apparues à partir des années quatre-vingts, consécutivement au phénomène d'externalisation opérées par de grandes firmes industrielles(12). Ce qui caractérise la relation de quasi-intégration, c'est d'abord le fait que la transaction n'est ni une pure transaction de marché, ni une transaction totalement internalisée au sein de la firme, puis la durabilité de la relation, et enfin la présence d'actifs spécifiques, que ceux-ci soient physiques ou huamins. Dès lors, la coordination ne s'effectue ni par le système des prix, ni par une autorité administrative -la firme-.

Or, il nous semble que ce concept de quasi-intégration industrielle peut être -en partie tout du moins- appliqué à la relation banque/entreprise. En effet, comme pour une relation de quasi-intégration industrielle, dans ce cas de figure, premièrement, la coordination ne se résume pas à l'établissement d'un contrat standard puisque l'information partagée est quantitiativement et qualitativement importante, dense, deuxièmement la relation se rigidifie, et troisièmement le crédit octroyé par la banque permet de financer des actifs relativement peu redéployables, autrement dit la quasiintégration autorise un investissement risqué. Une différence entre la quasiintégration industrielle et la quasi-intégration banque/entreprise réside toutefois dans la présence ou non, dans le cours de l'interaction, d'actifs spécifiques. Dans le cas de la quasi-intégration industrielle, et notamment de la quasi-intégration oblique, la présence d'actifs spécifiques, idiosyncrasiques, et la conception du produit en commun rapprochent les contractants de la situation qualifiée par Williamson d'"enfermement" (locked-in) (1985). En effet, ces actifs génèrent des coûts irrécouvrables (sunk costs) en cas de rupture de la relation, aussi bien pour le client que pour le fournisseur. En est-il de même dans le cas de la quasi-intégration banque/entreprise?

En fait, si dans une telle relation il n'existe pas d'actifs spécifiques physiques, des coûts irrécouvrables sont liés à la création, dans le cours de la relation conçue dans le long terme, d'une "information commune", information coûteuse et qui constitue le ciment de la relation et son efficience potentielle. Or, la sortie de la relation, pour la 
banque comme pour l'entreprise, signifie la perte de cette information commune, source de gains mutuels, et cette information s'assimile en partie à un actif idiosyncrasique, actif générant des coûts irrécouvrables en cas de rupture contractuelle. Dans ce cas, non seulement l'entreprise devra supporter les coûts d'information à acquérir pour la nouvelle banque, mais surtout sa volonté de changer de partenaire financier privilégié -les entreprises ont des relations avec plusieurs banques mais une seule joue le rôle de banque de premier rang- sera perçu dans la communauté comme un "signal d'alarme" défavorable (Harm, 1992). Dans une relation marchande, à l'opposé, comme nous l'avons noté, la sortie de la relation n'implique aucun coût dans la mesure où il n'existe pas d'information commune aux contractants.

D'un point de vue purement contractuel, si la structure de gouvernance par le marché repose sur un "sequential spot contract", la quasi-intégration se traduit par la passation, entre les agents, d'un contrat à long terme incomplet ("incomplete long term contract"). En effet, s'agissant d'une relation de long terme, les agents sont confrontés à la rationalité limitée. Autrement dit, il est ici impossible ex ante d'envisager tous les événements contingents susceptibles d'apparaître dans le cours de la relation. $\mathrm{Si}$, de plus, on conçoit que ces mêmes agents peuvent adopter des stratégies opportunistes dans le cours de la relation, alors on conçoit a priori qu'une relation de type quasi-intégration soit porteuse de risque et soit instable pour les contractants. C'est pour ces raisons que Williamson suggère que dans ces conditions, sur le marché des produits, l'intégration -la hiérarchie- se substitue à ce qu'il appelle la relation bilatérale client-fournisseur. En revanche, d'autres travaux, et notamment ceux de Aoki (1988), ont montré que lorsque que l'environnement est instable et la demande volatile, la quasi-intégration constitue une forme d'organisation susceptible de générer un cercle vertueux de coopération et de dégager une "quasi-rente".

Notre objectif, dans la suite de ce travail, consistera à montrer que la quasiintégration banque/PME est également une forme d'organisation supérieure en termes d'efficicence par rapport à la logique marchande et à la logique hiérarchique. Mais répondre à cette interrogation suppose l'analyse des formes de coordination qui soutiennent un tel type de relation.

\section{II/ Les formes de coordination dans la relation de quasi-intégration banque/entreprise : efficience interne et externe}

Si dans la relation marchande, la forme principale de coordination est clairement l'incitation contenue dans le contrat de courte période, dans une relation de type hiérarchique, comme nous l'avons vu, c'est l'autorité qui constitue la forme principale de coordination. En revanche, dans une relation de type quasi-intégration, il existe 
selon nous, comme dans la relation de quasi-intégration industrielle (Baudry, 1995) une pluralité de formes de coordination : I'autorité, l'incitation, et la confiance. Le tableau suivant récapitule cette démarche :

\section{La relation banque/entreprise : structures de gouvernance et formes de coordination}

\begin{tabular}{|c|c|c|c|}
\hline $\begin{array}{r}\text { structures de } \\
\text { gouvernance } \\
\begin{array}{c}\text { formes de } \\
\text { coordination }\end{array}\end{array}$ & Marché & $\begin{array}{c}\text { Quasi- } \\
\text { intégration }\end{array}$ & Hiérarchie \\
\hline Autorité & & $\mathbf{X}$ & $\mathbf{X}$ \\
\hline Incitation & $\mathbf{X}$ & $\mathbf{X}$ & \\
\hline Confiance & & $\mathbf{X}$ & \\
\hline
\end{tabular}

Après avoir réfléchit sur les formes de coordination présentes dans la relation de quasi-intégration, c'est-à-dire l'autorité, l'incitation et la confiance, nous serons alors en mesure d'identifier les avantages de cette structure de gouvernance, d'abord en termes d'efficacité interne à la relation $(A /)$, puis par rapport au contexte actuel de mutations bancaires $(\mathrm{B} /)$.

\section{A/ Les formes de coordination : autorité, incitation et confiance}

\section{1 / L'autorité}

L'autorité constitue la première forme de coordination dans une relation de type quasi-intégration. Comme nous l'avons souligné précédemment, dans ce cas de figure, une entreprise $\mathrm{Y}$ entretient une relation de long terme privilégiée avec une banque $\mathrm{X}$. En échange de cette relation privilégiée, la banque obtient un certain droit d'ingérence dans la gestion de l'entreprise. Autrement dit, l'entreprise $X$ accepte une certaine forme d'autorité qui peut d'ailleurs découler de droits de propriété, autrement dit d'une prise de participation de la banque dans le capital de l'entreprise. Cette forme d'autorité -affaiblit par rapport à celle qui résulterait d'un contrôle total de la banque sur l'entreprise (cas de la hiérarchie)- représente en quelque sorte la contrepartie du risque qu'accepte la banque en finançant des actifs peu redéployables (ce qui équivaut au fait que la garantie de la banque est plus faible que celle qui résulte de financement d'actifs facilement redéployables). En échange de cette prise de risque, les bailleurs de fonds obtiennent une forme de contrôle sur l'entreprise : ils ont des représentants au conseil d'administration, et ils partagent ainsi, d'une certaine manière, le pouvoir discrétionnaire normalement dévolu à la hiérarchie : droit de vote, discussions sur les choix de l'investissement de l'entreprise.

La différence profonde avec la forme que prend l'autorité dans la structure de gouvernance qualifiée de hiérarchie nous semble résider dans l'existence de contre- 
pouvoirs à la banque actionnaire-créancière. La quasi-intégration se caractérise ainsi par une relation de type partenariale entre les acteurs, porteuse d'un engagement des acteurs autour d'un projet commun.

L'autorité dans le modèle allemand de la banque maison est caractéristique d'un tel processus de quasi-intégration : le système financier allemand est construit sur la base de la banque universelle, ce qui signifie que la banque combine les fonctions de banque commerciale et de banque d'affaires.

La structure de financement du modèle allemand se distingue assez nettement de celle du modèle japonais, même si ces deux économies sont fondées sur le crédit. Ainsi, l'autofinancement tient une place essentielle dans l'économie allemande : $78 \%$ du financement brut des établissements non financiers entre 1986 et 1990 contre 54\% pour le Japon(13). En conséquence, si les relations financières avec les banques sont essentielles dans les deux économies, la dette intermédiée ne représente que $18 \%$ du financement brut des établissements non financiers allemands contre 32\% pour les entreprises japonaises. Cette moindre dépendance des entreprises allemandes à l'égard du crédit bancaire trouve aussi son origine dans le système même de la banque universelle : celle-ci est à l'interface entre les entreprises et les marchés financiers, les banques étant les premières maisons de courtage de valeurs mobilières.

La structure de propriété issue du modèle allemand montre une extrême concentration des actionnaires. Selon Prowse (1994), la participation moyenne des cinq plus gros porteurs est de plus de $40 \%$.

La relation d'autorité qui s'établit entre la banque et l'entreprise résulte des éléments précédents ainsi que de la structure de surveillance sous-jacente. Comme dans le système japonais, les banques sont amenées à surveiller - et à capter des informations - en tant que créancières et en tant qu'actionnaires. Le pouvoir de contrôle des banques est renforcé par le biais de l'utilisation des droits de vote de leurs déposants. II apparaît une étroite interaction entre les activités d'intermédiation financière des banques et leurs activités de crédit (Nardozzi, 1992). En effet, les informations captées soit en tant que fournisseurs de crédit - notamment sur la continuité des paiements d'intérêts - soit en tant que porteurs de titres, renforcent mutellement les deux activités de la banque universelle(14).

La structure de surveillance est constitutive de la gestion participative à l'allemande. Deux organes de gestion sont à considérer (OCDE, 1994). Le premier est le Directoire dont le mandat est de 5 ans. Il a un pouvoir exécutif et a l'autorité sur la gestion de l'entreprise au jour le jour. Le second organe de gestion est le Conseil de Surveillance. Ce dernier se réunit 2 à 4 fois par an. Son importance résulte de son rôle d'observateur de l'évaluation de l'évolution de l'entreprise. L'influence des banques s'exprime dans ce Conseil de Surveillance. Cependant, et ceci est essentiel, il existe des 
contre-pouvoirs à la banque universelle. Ce sont les deux autres participants à ces organes de gestion : les dirigeants, mais surtout les salariés. Dès lors, le système de surveillance et la relation d'autorité qui en découle se fondent d'abord sur la négociation et la coopération, et moins sur la sanction.

\section{2/ L'incitation}

L'incitation a pour objet premier l'établissement d'un certain nombre de procédures et de règles susceptibles d'exercer une influence sur le comportement et les stratégies des acteurs. L'incitation a ainsi une double perspective :

- inciter les acteurs à trouver des solutions coopératives, surmontant par làmême les situations sous-optimales du type dilemme du prisonnier. A ce niveau, l'incitation vise à modifier le comportement stratégique des acteurs.

- assurer une transmission de l'information telle que les acteurs "ennemis" et "amis" - pour reprendre les expressions de JSobel (1989) - puissent se révéler. Dans le cadre de la théorie de l'agence, cela signifie que le principal est dans l'incertitude quant aux intentions effectives de l'agent.

Dans cette dernière hypothèse, la relation de prêt implique pour la banque d'élaborer dans une situation d'incertitude des procédures d'incitation telles que les pertes liées aux emprunteurs défaillants (les ennemis) soient minimisées.

Les modèles d'incitation à $n$ périodes fournissent une première approximation intéressante pour rendre compte de l'importance de la durée de la relation. Diamond (1989) décrit un modèle d'incitation par l'effet-réputation. En raison d'un processus d'apprentissage au cours du temps, la longueur de l'horizon temporel est un élément essentiel de la relation de prêt. Plus précisément, une longue période de non défaut à des effets bénéfiques pour le débiteur de par la réduction de ses charges de remboursement. Nous avons ainsi les relations suivantes:

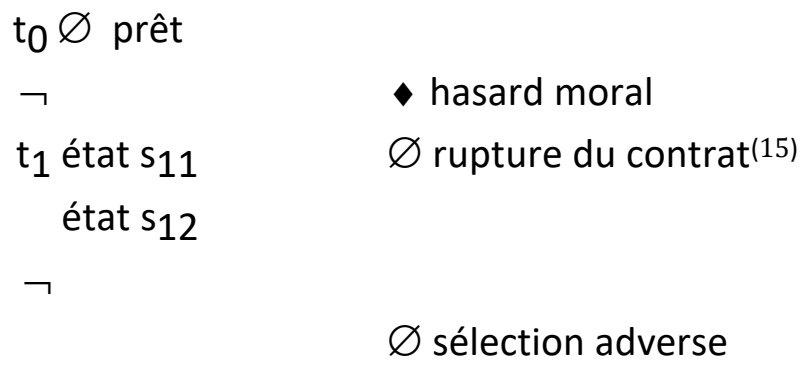

$\varnothing$ sélection adverse

$t_{2}$ nouveau prêt

L'antisélection est liée au fait que les nouveaux emprunteurs font face à des effets incitatifs plus importants par rapport aux anciens emprunteurs. Dans ce contexte, "la valeur d'une bonne réputation s'accroît sur le temps"(16). L'enseignement 
majeur de ce modèle est le suivant : de par la liaison entre les conditions d'emprunts et le prolongement sur plusieurs périodes d'une situation de non défaut, les entreprises sont incitées à respecter les engagements contractés auprès des banques. Autrement dit, le respect des engagements a une valeur monétaire qui influence la fonction de préférence de l'emprunteur. Cet effet induit peut lui-même se décomposer en deux éléments distincts, à savoir un effet induit interne - lorsque les conditions de prêts sont modifiées avec le même partenaire financier - et un effet induit externe - lorsque l'emprunteur utilise cette réputation pour se signaler auprès d'autres bailleurs de fonds. La remise en cause de la réputation constitue un coûts irrécouvrable et nécessite en outre une nouvelle période d'investissement pour la firme.

Le modèle précédent, s'il constitue un apport important dans l'appréhension de la relation banque-entreprise, souffre néanmoins d'une limite : les interactions entre la firme et la banque n'apparaissent pas nettement. Pour les faire apparaître, il est nécessaire de considérer les relations qui s'établissent durant le déroulement du contrat : c'est l'analyse ongoing.

Les analyses de Hellwig (1989 et 1990) et Von Thadden (1990) constituent sans aucun doute la pierre angulaire de ce type d'approche. Le point de départ est le suivant : "la nature de long terme des relations financières change la nature des problèmes d'incitation"(17). En effet, le contrat d'incitation optimal est "statedependent payment". II en résulte deux implications importantes :

- des mécanismes d'exécution (enforcement mechanism) doivent être mise en place pour éviter les comportements opportunistes du type renégociation intempestive du contrat ou recherche d'autres financeurs après avoir bénéficié des apports avantageux de la banque ;

- dans la mesure où les contrats sont rarement complets, un système de règles doit être élaboré, dont l'objectif est de construire un common knowledge. Dans le système allemand, l'une des règles essentielles est la restructuration de l'entreprise sous la responsabilité de la banque maison selon un processus de co-détermination avec les représentants des salariés(18).

Dans son modèle, Von Thadden étudie les conséquences du monitoring qui apparaît après la signature du contrat, mais avant sa fin. Il se situe donc dans l'analyse ongoing, entre l'ex ante et l'ex post. Les principales caractéristiques du modèle sont les suivantes : il y a deux périodes d'investissement, deux types d'entreprises (les bonnes et les mauvaises) et deux stratégies d'investissement (court terme et long terme). A la fin de la période 1 , les résultats obtenus par les entreprises permettent d'établir une distinction entre les firmes. Pour cela, les banques disposent de deux sources d'informations : l'observation des rendements et le monitoring au cours du contrat. 
L'apport de la relation ongoing apparaît ici. En effet, en l'absence d'un tel monitoring, la simple observation des rendements conduit à supprimer les prêts dès la fin de la première période. Un tel comportement conduit les firmes à élaborer des stratégies d'investissement à court terme, privilégiant le résultat de la première période. A contrario, le monitoring ongoing fournit à la banque des informations supplémentaires sur la firme qui sont susceptibles de lui permettre d'identifier les bonnes firmes, et ce même si les résultats de la première période sont décevants. Les conditions de financement de la seconde période pèsent alors moins sur les décisions des entrepreneurs. En conséquence, la relation de prêt perdure et les entreprises sont incitées à mener des stratégies d'investissement à long terme.

La relation banque/entreprise est ainsi fondée sur un processus "d'interaction stratégique" dans lequel "the notion of 'optimal' behaviour must be replaced by a notion of 'equilibrium' behaviour"(19). Les règles permettent de réaliser une certaine convergence des anticipations des acteurs et incitent par là-même à la coopération.

\section{$\underline{3 / \text { La confiance }}$}

La confiance constitue la troisième forme de coordination dans une relation de type quasi-intégration. Dès lors que les agents sont amenés à conclure des contrats incomplets, la confiance est indispensable pour soutenir l'échange. Mais, contrairement à certaines analyses ${ }^{20}$, nous ne pensons pas que la confiance puisse constituer une forme de coordination indépendante, c'est-à-dire nécessaire et suffisante pour générer la coopération dans une relation de quasi-intégration.

D'une certaine manière, la confiance est nécessaire dès lors qu'il existe une incertitude associée à l'échange. Pour Gambetta (1988, p. 217), la confiance se définit comme "un niveau particulier de probabilité subjective avec lequel un agent évalue qu'un autre agent ou un groupe d'agents va produire une action particulière, avant qu'il puisse évaluer cette action (indépendamment de sa capacité d'être en mesure de l'évaluer), dans un contexte où sa propre action en est affectée ". Autrement dit, pour Gambetta, la confiance se constitue dans des conditions d'incertitude sur l'action des autres, et sa première propriété est de réduire l'incertitude sur le comportement futur de l'autre. Mais la confiance constitue également une réponse à l'apparition d'évènements exogènes, c'est-à-dire des événements non anticipés au départ de la relation (en $t_{0}$ ), donc en situation d'incomplétude contractuelle. La confiance va alors permettre de remplir "les blancs" du contrat explicite. Comme l'indique Luhmann (1979), la confiance permet de faire face à la complexité et de réduire l'incertitude.

Quels sont alors les dispositifs concrets qui permettent de soutenir la confiance ? Pour nous, deux dispositifs remplissent cette fonction : le contexte social et les relations interpersonnelles. 
L'analyse des districts industriels et des "systèmes industriels localisés" met en avant l'importance des normes d'obligation et de coopération dans le processus de l'échange. Pour Saglio (1991), les échanges qui s'établissent entre des firmes membres d'un même réseau industriel conduisent à des "systèmes de choix préférentiels", et, par le biais de croyances et de valeurs communes, l'échange économique se transforme en "échange social". II en est de même dans la relation de quasiintégration banque/entreprise. La proximité géographique des contractants et donc le sentiment d'appartenance à une même communauté permet de produire de la confiance et de soutenir la transaction. C'est ainsi qu'une banque qui entretient des rapports étroits et à long terme avec des entreprises de la région n'aura pas le même comportement qu'avec des clients plus lointains. D'une certaine manière, des normes d'obligation et de coopération sont présentes dans la relation de quasi-intégration, normes qui contraignent l'action des agents et qui génèrent des comportements a priori non rationnels d'un point de vue économique.

Les relations interpersonnelles produisent également de la confiance. Pour Granovetter (1985), ce ne sont pas les caractéristiques des personnes qui importent, mais les relations que les personnes entretiennent entre elles. Contrairement aux néoinstitutionnalistes, l'argument de l'encastrement ("embeddedness") insiste au contraire sur le rôle des relations personnelles concrètes et des structures de telles relations pour générer la confiance et décourager la malhonnêteté.

Dans une structure de gouvernance marchande, les relations sont totalement impersonnelles. Autrement dit, ce n'est pas la qualité personnelle de celui qui contracte qui importe, mais les actifs industriels que cette personne est susceptible d'apporter. Au contraire, dans une relation de quasi-intégration, la qualité propre des personnes importe. II existe un processus interactif de communication, un transfert d'informations dense entre des personnes qui se connaissent et qui tissent progressivement des liens de confiance, dans une inscription intertemporelle. Autrement dit, dans le cours de l'interaction, des actifs spécifiques humains se constituent, actifs qui vont supporter la transaction. En définitive, et toujours pour reprendre la terminologie d'Hirschman (1972), si le marché se caractérise par l'exit, la quasi-integration se situe nettement du côté de la voice.

\section{B/ Pluralité des formes de coordination et efficience}

\section{$\underline{1 / \text { L'efficience interne }}$}

Les développements qui précèdent montrent que dans une relation de quasiintégration, l'échange économique caractéristique d'une relation marchande se transforme bien en échange social. Or, dans l'échange social intervient un système de 
contreparties entre les "transactants" qui n'est pas inscrit explicitement au contrat. Dans une relation de type quasi-intégration, l'échange social fait par exemple appel à la notion d'engagement, de mobilisation, qui repose sur la confiance, contrairement à l'échange économique dans lequel les obligations des uns et des autres sont entièrement spécifiées à l'avance (existence d'un contrat contingent complet). Du fait de l'incomplétude contractuelle intervient dans le cours de la transaction le phénomène de "réciprocité" : le contrat conclu ex ante repose alors en partie sur des promesses inscrites implicitement au contrat, promesses qui vont s'échanger dans le temps. En ce sens, la confiance constitue une réponse à l'apparition d'évènements exogènes et endogènes- non prévus au départ de la relation, lorsque celle-ci est de longue durée. Supposons par exemple que l'entreprise connaisse momentanément des difficultés financières, consécutivement à une diminution de la demande finale évènement exogène non prévu ex ante-. La banque peut alors décider d'adopter un comportement opportuniste (stratégie non coopérative) en refusant d'aider l'entreprise à surmonter ses difficultés passagères. Mais, adopter une telle stratégie conduirait la banque à encourir des pertes liées aux affaires futures qu'elle est amenée à avoir avec l'entreprise. Ce serait détruire le capital de confiance, l'information commune et les effets bénéfiques issus de la relation conçue dans le long terme.

Il en est de même en cas d'irruption d'évènements endogènes : une entreprise qui a effectué des mauvais choix d'investissement bénéficiera éventuellement d'une aide de la banque. En ce sens, la banque joue auprès de l'entreprise un rôle d'assureur vis-à-vis des risques encourus en cas d'investissement risqué de la part de l'entreprise. Bien évidemment, en retour, la banque attend de l'entreprise des efforts pour remédier aux difficultés temporaires et elle appliquera des taux d'intérêt effectifs relativement plus élevés lors de la renégociation du contrat. D'ailleurs, l'entreprise usera éventuellement de l'autorité dont elle dispose pour mener à bien le redressement nécessaire.

Par ailleurs, l'autorité, librement acceptée par l'entreprise, permet de compléter l'information obtenue par le canal de la confiance ${ }^{21}$, et donc de remédier au problème de l'antisélection. Ce type de relation banque/entreprise a deux avantages principaux du point de vue de la dynamique industrielle d'une économie. D'une part, l'étroitesse des liens qui se tissent entre les acteurs permet d'asseoir les relations dans la durée, et les entreprises sont alors mieux à même de s'engager dans des projets dont les perspectives de retour sur investissement sont lointaines. La prise de contrôle par la banque et la confiance existante entre les acteurs constituent d'ailleurs une garantie d'une solution productive en cas de difficultés trop importantes. D'un point de vue empirique, certaines études montrent par exemple que les banques japonaises et 
allemandes "organisent le sauvetage de l'entreprise, en apportant des capitaux supplémentaires ainsi que leur savoir-faire" (Rivaud-Danset et Salais, 1992). D'autre part, la relation banque/entreprise assise sur l'autorité et la confiance permet une amélioration substantielle de la liquidité de la firme. Ainsi, Hoshi et alii (1990) ont montré dans leur étude que la contrainte de liquidité interne est relaxée lorsque la banque entretient d'étroites relations avec l'entreprise qui a des projets d'investissement(22). II s'agit-là d'un facteur de flexibilité financière qui autorise un dynamisme certain en matière d'investissements.

Pour autant, la confiance que la banque entretient avec l'entreprise ne saurait constituer une forme de coordination suffisante car l'entreprise est toujours en mesure d'adopter un comportement opportuniste, autrement dit elle peut être tentée d'abuser de cette confiance. En ce sens, la confiance constitue un dispositif fragile pour soutenir la coordination. C'est pour cette raison que l'incitation, troisième forme de coordination, constitue une force de rappel pour l'entreprise. Les dispositifs incitatifs garantissent à la banque un certain niveau d'effort de la part de l'entreprise, donc permettent à la banque de faire face au risque moral, et assurent également une transmission de l'information. Comme on le constate, autorité, incitation et confiance sont indissociables dans la relation de quasi-intégration et ne sont pas contradictoires. 


\section{2/ Mutations bancaires et efficience externe}

Les discussions relatives à l'alternative système financier fondé sur le marché versus fondé sur le crédit tendent à insister sur la réversibilité à court terme de la relation qui apparaît comme un déterminant essentiel du recours au modèle marchand lorsque l'environnement est très instable. A contrario, la relative rigidité de la relation banque-entreprise dans le modèle de quasi-intégration semblerait interdire des modifications rapides, radicales et fréquentes lorsque l'environnement ne s'est pas stabilisé(23). En conséquence, face à l'environnement turbulent qui caractérise la situation actuelle, la structure de gouvernance fondée sur la quasi-intégration paraît devoir être remise en cause. Cependant, si l'on prend en compte les conséquences des mutations financières, nous sommes amenés à nuancer cette affirmation. Loin d'être une structure qui rigidifie les relations de financement, la quasi-intégration nous paraît faire preuve d'une efficience externe.

Les mutations financières engagées depuis le début des années 80 dans les principaux pays développés revêtent trois caractéristiques principales (OCDE, 1994). Premièrement, la dérèglementation a engendré un rôle croissant des mécanismes de marché lors de la détermination des choix de placement et des sources de financement. Deuxièmement, les innovations financières ont entraîné un élargissement considérable de la gamme des instruments de financement, notamment du point de vue de leur négociabilité qui s'est accrue. Une telle amélioration de la négociabilité "a une contrepartie, celle de soumettre une part croissante des décisions d'engagement du capital dans l'industrie au jugement collectif des acteurs des marchés financiers"(24). Troisièmement enfin, l'Etat s'est progressivement désengagé des multiples procédures de financement de l'économie, suite d'ailleurs au relatif échec de son intervention.

Les conséquences de ces mutations financières sont multiples. Deux nous intéressent plus particulièrement dans le cadre de la structure de gouvernance quasiintégration. D'une part, en raison de l'accroissement des considérations liées au marché, les capitaux ont été alloués différemment entre les types d'investissement. Ainsi, les investissements qui ne peuvent faire l'objet d'une procédure de sécuritisation en raison de leur spécificité et ceux qui ne peuvent engendrer une contrepartie matérielle rapide tendent à trouver des conditions de financement plus difficiles. Au premier chef de ces investissements se trouvent les processus innovants(25). D'autre part, si les grandes entreprises ont pu bénéficier de l'élargissement de la gamme des instruments de financement, les PME-PMI sont demeurées assez largement à l'écart de ces innovations. II en résulte un processus de dualisation des conditions de financement qui rend plus difficile le financement externe des PME-PMI. 
C'est à ce niveau que la quasi-intégration peut représenter un financement alternatif efficient. Une telle proposition trouve ses origines dans la situation générale des PME-PMI dans un contexte de généralisation de la banque à l'acte, i.e. de la structure de gouvernance marchande.

Premièrement, les mutations financières n'ont pas mis fin à la contrainte endémique des $\mathrm{PME}$, à savoir leur capacité à engendrer des fonds propres. La faiblesse des fonds propres des PME contraint directement l'accès au financement bancaire. En effet, le faible niveau des fonds propres est interprété comme un signal négatif puisque synonyme d'une moindre prise de responsabilité de la part de l'entrepreneur-dirigeant. En outre, il apparaît une fragilisation de la situation des entreprises du point de vue de la contrainte de solvabilité.

Secondement, la contrainte de production d'information des PME-PMI est très élevée par rapport aux grandes entreprises. Le défaut d'accès aux marchés financiers producteurs d'informations publiques et l'absence d'agences de rating spécialisées dans I'appréciation des situations financières des PME-PMI représentent les facteurs essentiels de l'existence d'une telle contrainte. Or, la forme de coordination marchande repose essentiellement sur l'existence d'une information produite sur les marchés. Dès lors, la contrainte d'information des PME-PMI renforce les probabilités d'occurence des comportements opportunistes et de défiance. La banque n'est pas incitée à rechercher de l'information rare et donc coûteuse, et ceci conduit les intermédiaires financiers à être réticents à s'engager auprès des PME (Binks et alii, 1992).

Face à ces deux contraintes structurelles, la structure de gouvernance fondée sur la quasi-intégration fournit un certain nombre de réponses. Cette efficience externe trouve ses origines dans la combinaison des formes de coordination. Tout d'abord, la stratégie d'engagement réciproque des partenaires les incite à avoir une démarche coopérative propice au dépassement des obstacles purement marchands. La confiance joue ici un rôle essentiel dans la pérennité de la relation. Ensuite, la double caractéristique de la banque - actionnaire et créancière - a un double avantage du point de vue de l'incitation et de l'autorité :

- inciter les PME à renforcer leurs fonds propres par l'émission de titres de propriété ;

- conduire les banques à une meilleure connaissance des entreprises de par l'accès et à la production d'informations, ce qui à terme doit conduire à une réduction des coûts des emprunts de part un controle plus étroit des emprunteurs.

Enfin, et ceci nous paraît essentiel, la quasi-intégration peut éviter la dépendance subie des PME à l'égard du financement bancaire dans la mesure où, ce 
qui prévaut, c'est la relation partenariale, et non la relation marchande ou la relation hiérarchique.

\section{Conclusion}

Notre tentative de rapprochement entre la relation banque-entreprise et la relation interentreprises s'avère en définitive féconde puisqu'en effet, dans les deux cas, les structures de gouvernance sont comparables. Nous avons notamment montré que dans les deux cas, la quasi-intégration repose sur une pluralité de formes de coordination, c'est-à-dire l'autorité, l'incitation et la confiance. Or, il semble bien que dans la période actuelle caractérisée par une incertitude relativement élevée et par le processus de globalisation financière, la quasi-intégration banque-entreprise, comme la quasi-intégration industrielle, constitue la structure de gouvernance pertinente. S'il en est ainsi, c'est parce que les formes de coordination se combinent et s'épaulent mutuellement.

Néanmoins, il ne faut pas en conclure que la quasi-intégration constituerait une forme d'organisation qui serait intrinsèquement supérieure aux deux autres structures de gouvernance, le marché et la hiérarchie. En effet, un retour à une croissance régulière et/ou une stabilisation de l'environnement institutionnel pourrait signifier un retour vers un fonctionnement plus marchand. 


\section{Notes}

(1) Nous prolongeons en ce sens l'analyse de C. Mayer (1990) selon laquelle - dans l'ensemble des pays développés - les rétentions de profit sont la source principale de financement. En conséquence, le recours à la quasi-intégration doit trouver son explication non pas seulement dans un quelconque besoin de financement mais plus fondamentalement dans la circulation de l'information entre les acteurs.

(2) O.E. Williamson (1975), p.64.

(3) L'alternative défaut/non défaut est présente dans le modèle de J. Stiglitz et A. Weiss (1983) tandis que l'alternative faillite/non faillite est propre au modèle de D. Gale et M. Hellwig (1985).

(4) En ce sens, introduire la relation de clientèle dans ces modèles nous paraît être un extension implicite de leurs enseignements sans qu'ils en rendent explicitement compte. La logique de l'exit reste prédominante.

(5) J.E Stiglitz et A. Weiss (1983), p.912.

(6) F. Allen (1983), p.640.

(7) Il serait également possible d'envisager le cas de figure inverse, c'est-à-dire la prise de contrôle de la banque par l'entreprise.

(8) S. Prowse (1994), p.132.

(9) L'OCDE (1994) montre les difficultés du système japonais à exploiter les opportunités technologiques et commerciales dans quelques secteurs, notamment les biotechnologies et les logiciels.

(10) Pour une présentation générale de ces mutations, voir P. Geoffron et M. Rubinstein (1994). Cependant, il convient de relativiser l'ampleur de ces mutations dans le cadre du système de la banque principale. En effet, comme le souligne T. Hoshi (1994), c'est désormais par les participations croisées que semble d'abord s'exprimer ce système qui ne semble donc pas fondamentalement remis en cause dans sa nature.

(11) J. Houssiaux (1957), p.222.

(12) Cf. notamment les travaux de M. Aoki pour le Japon (1988), et B. Baudry pour la France (1995).

(13) Selon les données de S. Prowse (1994).

(14) Cependant, en dépit de cette position privilégiée, il ne semble pas en résulter une distorsion dans la concurrence. Sur ce point, voit G.J. Benston (1994).

(15) Avec $\mathrm{s}_{11}=$ situation de défaut et refus de prêt $/ \mathrm{s}_{12}=$ situation de non défaut et nouveau contrat.

(16) D.W. Diamond (1989), p.831.

(17) M. Hellwig (1989), p.280.

(18) En apparence, le processus de restructuration n'est donc pas très différent par rapport à celui du modèle japonais. La sanction ultime de la défaillance est effectivement similaire, mais la co-détermination dans le système allemand permet à la relation de hiérarchie de ne pas jouer. Cette proximité entre les deux modèles montre par contre que l'étroitesse des relations banque-entreprise, et leur inscription dans la durée, impliquent de définir précisément des règles permettant d'encadrer les comportements des acteurs.

(19) M. Hellwig (1989), p.283.

(20) Nous faisons ici référence à l'analyse de D. Rivaud-Danset et R. Salais (1992) en termes de convention de financement. Ces auteurs dégagent ainsi une convention de financement interpersonnelle dans laquelle la confiance, à travers la singularité des personnes, constitue le seul dispositif de coordination.

(21) On retrouve ici d'une certaine façon la "logique domestique" analysée par L. Boltanski et L. Thévenot (1991).

(22) Les auteurs apprécient la liquidité interne en retenant deux éléments : par le cash flow courant dégagé et par le stock de titres négociables détenus par les firmes.

(23) Cf. notamment E. Berglof (1990) et J.L. Christensen (1992).

(24) OCDE (1994), p.43.

(25) Voir J.P. Allegret (1995) pour une analyse des investissements innovants. 


\section{BIBLIOGRAPHIE}

AGHION P. et P. BOLTON [1992], "An incomplete contracts approach to financial contracting", Review of Economic Studies, vol.52, pp.473-494.

AKERLOF G. [1970], "The market for "lemons" : qualitative uncertainty and the market mechanism", The Quaterly Journal of Economics, vol. 84, pp. 488-500.

ALLEGRET J.P. [1995], "Le financement des PME innovantes : sous quelles conditions les technopoles peuvent-elles constituer une réponse adéquate?", à paraître dans Revue Internationale PME.

ALLEN F. [1983], "Credit rationing and payment incentives", Review of Economic Studies, vol.50, pp.639-646.

ALLEN F. [1993], "Stock markets and ressource allocation, C. Mayer et X. Vives (eds), Capital markets and financial intermediation, Cambridge, Cambridge University Press, pp.81-108.

AOKI M. [1988], Information, incentives, and bargaining in the Japanese Economy, Cambridge, Cambridge University Press.

AOKI M. et G. DOSI [1992], "Corporate organization, finance and innovation", V. Zamagni (ed.), Finance and enterprise, Londres, ed. Harcourt Brace Jovanovich, pp.3761.

BAUDRY B. [1995], L'économie des relations interentreprises, Paris, Repères, La Découverte.

BENSTON G.J. [1994], "Universal banking", Journal of Economic Perspectives, vol.8, $\mathrm{n}^{\circ} 3$, pp.121-143.

BERGLOF E. [1990], "Capital structure as a mechanism of control : a comparison of financial systems", M. Aoki, B. Gustafsson et O.E. Williamson (eds), The firm as a nexus of treaties, Londres, Sage Publications, pp.237-262.

BINKS M.R., C.T. ENNEW et G.V. REED [1992], "Information asymmetries and the provision of finance to small firms", International Small Business Journal, vol.11, $\mathrm{n}^{\circ} 1$, pp.35-46.

BOLTANSKI L. et L. THEVENOT [1991], De la justification; Les économies de la grandeur, Paris, Gallimard.

CHRISTENSEN J.L. [1992], "The role of finance in national systems of innovation", B.A. Lundvall (ed.), National systems of innovation, Londres, Pinter Publishers, pp.146-168.

DIAMOND D.W. [1989], "Reputation acquisition in debt markets", Journal of Political Economy, vol.97, $n^{\circ} 4$, pp.828-862.

GALE D. et M. HELLWIG [1985], "Incentive-compatible debt contracts I : one-period problem", Review of Economic Studies, vol.52, October, pp.647-664.

GAMBETTA D [1988], "Can we trust trust ?", D. Gambetta (ed.), Trust : making and breaking cooperative relations, Oxford, Blackwell, pp. 213-237.

GEOFFRON P. et M. RUBINSTEIN [1994], "Les mutations du modèle japonais de Corporate governance", Revue d'Economie Financière, n³1, Hiver, pp.225-235.

GERTNER R.H., J.C. STEIN et D.S. SCHARFSTEIN [1994], "Internal versus external capital markets", Working Paper, National Bureau of Economic Research, $n^{\circ} 4776$, June. 
GOODHART C.A.E. [1994], "Banks and the control of corporations", Economic Notes, vol.23, n¹, pp.1-18.

GORTON G. et J. KAHN [1993], "The design of bank loan contracts, collateral, and renegotiation", Working Paper, National Bureau of Economic Research, $n^{\circ} 4273$, February.

GRANOVETTER M. [1985], "Economic action and social structure : the problem of embeddedness", American Journal of Sociology, vol. 91, n 3, pp. 481-510.

HARM C. [1992], "The financing of small firms in Germany", Policy ResearchFinancial Policy and Systems, World Bank Working Papers, $n^{\circ} 899$.

HAUBRICH J.G. [1989], "Financial intermediation : delegated monitoring and longterm relationships", Journal of Banking and Finance, vol.13, pp.9-20.

HELLWIG M. [1989], "Asymmetric information, financial markets, and financial institutions - where are currently going ?", European Economic Review, vol.33, pp.277-285.

HELLWIG M. [1990], "Banking, financial intermediation and corporate finance", A. Giovannini et C. Mayer (eds), European financial integration, Londres, CEPR, pp.35-63.

HIRSCHMAN A. O. [1972], Face au déclin des entreprises et des institutions, Paris, Les éditions ouvrières.

HOSHI T. [1994], "The economic role of corporate grouping and the main bank system", M. Aoki et R. Dore (eds), The Japanese firm, the sources of competitive strength, Oxford, Oxford University Press, pp.285-309.

HOSHI T., A. KASHYAP et D. SCHARFSTEIN [1990], "Bank monitoring and investment : evidence from the changing structure of Japanese corporate banking relationships", R.G. Hubbard (ed.), Asymmetric information, corporate finance and investment, National Bureau of Economic Research, Chicago, The University of Chicago Press, pp.105-126.

HOUSSIAUX J. [1957], "Le concept de quasi-intégration et le rôle des sous-traitants de l'industrie", Revue Economique, Mars, pp. 221-247.

LUHMANN N. [1979], Trust and power, New YorkJohn Wiley and Sons.

MAYER C. [1990], "Financial systems, corporate finance, and economic development", R.G. Hubbard (ed.), Asymmetric information, corporate finance and investment, National Bureau of Economic Research, Chicago, The University of Chicago Press, pp.307-332.

NARDOZZI G. [1992], "National specificities and current tendencies in financial systems", V. Zamagni (ed.), Finance and enterprise, Londres, ed. Harcourt Brace Jovanovich, pp.247-263.

OCDE [1994], Les systèmes nationaux de financement de l'innovation, Mars, Paris.

PROWSE S. [1994], "Corporate governance : comparaison internationale", Revue d'Economie Financière, $n^{\circ} 31$, Hiver, pp.119-158.

RIVAUD-DANSET D. [1991], "La relation banque-entreprise, une approche comparée", Revue d'Economie Financière, $n^{\circ} 16$, Mars.

RIVAUD-DANSET D. et R. SALAIS [1992], "Les conventions de financement des entreprises. Premières approches théoriques et empiriques", Revue Française d'Economie, pp.81-120.

SAGLIO J. [1991], "Echange social et identité collective dans les systèmes industriels", Sociologie du Travail, vol.33, n4, pp. 529-544. 
SOBLE J. [1985], "A theory of credibility", Review of Economic Studies, vol.52, pp.557-573.

STIGLITZ J.E. [1985], "Credit markets and the control of capital", Journal of Money, Credit and Banking, vol.17, n², pp.133-152.

STIGLITZ J.E. [1987], "The causes and consequences of the dependence of quality on price", Journal of Economic Literature, Mars, pp.1-48.

STIGLITZ J.E. et A. WEISS [1983], "Incentive effects of termination : application to the credit and labor markets", American Economic Review, vol.73, pp.912-927.

VON THADDEN E.L. [1990], "Bank finance and long-term investment", Working Paper, Universitad Basel December.

WEBB D.C. [1991], "Long-term financial contracts can mitigate the adverse selection problem in project financing", International Economic Review, vol.32, $\mathrm{n}^{\circ} 2$, pp.305-320.

WILLIAMSON O.E. [1975], Markets and hierarchies : analysis and anti-trust implications, New York, Free Press.

WILLIAMSON O.E. [1985], The economic institutions of capitalism, New York, Free Press.

WILLIAMSON O.E. [1988], "Corporate finance and corporate governance" Journal of Finance, vol.43, n³, pp. 567-591. 